\title{
A new detection method of power quality disturbance signal
}

\author{
Song Changpo ${ }^{1}$, Bai Jie ${ }^{1}$, Shen Yan ${ }^{1}$, Wang Tianzheng \& Lu Shan ${ }^{2}$ \\ Power System Stability Control Company, NARI-TECH, Nanjing, China ${ }^{1}$ \\ State Grid Shanxi Electric Power Research Institute, Taiyuan, China ${ }^{2}$ \\ E-mail: songchangpo@sgepri.sgcc.com.cn E-mail: Is8760033@163.com
}

\begin{abstract}
Keywords: power quality disturbance; detection and location; S-transform; disturbance localization algorithm
\end{abstract}

\begin{abstract}
For the detection and location of power quality disturbance signal problem, a disturbance localization algorithm is proposed based on the mean sum of the square of S-transform amplitude, with this method the time of occurrence and duration of the disturbance signal is simulated. Simulation results show that the proposed algorithm is simple and effective, able to accurately analyze the disturbance signal, and the magnitude of voltage perturbation, voltage sag and voltage swell can be estimated.
\end{abstract}

\section{Introduction}

Widespread use of power electronic devices and other non-linear loads, bring to the grid power quality problems, such as harmonics, voltage swells, voltage sags, voltage interruptions and other high-frequency transient disturbances. The solution to these problems will depend on a variety of timely and accurate disturbance signal detection, location and classification. Currently, most systems use a Fourier transform, dq transform or wavelet transform to detect disturbances and extracting features, then the use of neural networks to automatically identify quality disturbances. Fourier transform part of the global transformation, were only able to analyze the time domain or frequency domain signal, and cannot be analyzed for non-stationary signals that have transient, mutation and other characteristics ${ }^{[1]}$. The wavelet transform is a time-frequency analysis method, because it has a good time domain and frequency domain localization properties, suitable for the analysis of transient signals and transient signals, has been widely used in power system ${ }^{[2]}$. However, as described in the literature, in the detection of high-frequency disturbances, the wavelet transform has a great advantage, for the detection of low frequency disturbances such as voltage sags, etc., but not a good detection and localization. Especially in noisy environments, using wavelet transform becomes complex, the lack of intuitive results ${ }^{[3]}$.

Stransform (S-Transform) by Stock Well et al in 1996 first proposed as an extension of the wavelet transform, $\mathrm{S}$ transform gradually attracted people's interest, and has been applied to the detection and recognition of power quality disturbances ${ }^{[4]}$. In this paper, based on S-transform perturbation amplitude and mean square localization algorithm by MATLAB simulation, comprehensive study of the algorithm in detection, localization accuracy when a variety of power quality disturbances such as harmonics, voltage sags, voltage swells, voltage interruptions and voltage flicker.

\section{The basic principle of S-transform}

If the signal is a continuous signal, which is a continuous one-dimensional S-transformation is defined as follows:

$$
S(\tau, t)=\int_{-\infty}^{\infty} s(t) w(\tau-\mathrm{t}) \exp (-i 2 \pi f t) d t
$$

And, 


$$
\left.w(\tau-t)=|f| / \sqrt{2 \pi} \exp ^{-}-f(\tau-t)^{2} / 2\right]
$$

In the formula (1), (2), w( $\tau-t)$ is a Gaussian window, $\tau$ is the time axis Gaussian window parameter position, $f$ is frequency.

In this equation, $\mathrm{S}$ transform function must satisfy the following normalization condition, namely

$$
\left.\int_{-\infty}^{\infty}|f| / \sqrt{2 \pi} \exp ^{-} f^{2}(\tau-t)^{2} / 2\right] d \tau=1
$$

Then, S-transform eventually be written

$$
S(\tau, t)=\int_{-\infty}^{\infty} s(t)|f| / \sqrt{2 \pi} \exp ^{\left.-f^{2}(\tau-t)^{2} / 2\right] d t}
$$

$|f| / \sqrt{2 \pi} \exp \left[^{-f^{2}(\tau-t)^{2}} / 2\right.$ is a Gaussian window, through changes in the value of $\tau$, and to control the parameters of the Gaussian window in the timeline position. $f$ represents frequency, by varying $f$, achievable changes Gaussian window. So you could easily analyze a high-frequency signal or a lowfrequency signal ${ }^{[5]}$. As can be seen from the formula, $S$ transform and short time Fourier transform is not the same, except that the height and width of the Gaussian window varies with frequency, thus overcoming the shortcomings of the Fourier transform of the window height and width cannot change. Continuous one-dimensional inverse S-transform as follows:

$$
s(t)=\int_{-\infty}^{\infty}\left[\int_{-\infty}^{\infty} S(\tau, t) d \tau\right] \exp (i 2 \pi f t) d f
$$

If the $\mathrm{S}$ transform divided by $\exp (i 2 \pi f t)$, that becomes a signal of the wavelet transform, Therefore, S-transform can be seen as a "phase correction" continuous wavelet transform, and can be derived from the continuous wavelet transform. If the mother wavelet is defined as a Gaussian window function, and a product of a complex vector, and then by continuous wavelet formula, you can get $S$ transform. If the formula (1) $w(\tau-t)$ and $s(t) \exp (-i 2 \pi f t)$, respectively, as an independent, the $S$ transformation can be seen from the Fourier transform of the convolution ${ }^{[6]}$. Thus, $\mathrm{S}$ transform algorithms can change fast Fourier transform algorithm implementation. This is a disguised form of realization of S-transform.

\section{Discrete processing of S transform}

In practical applications, the signal acquisition to input to the processor, is already discrete signal, the signal to be processed by S transform are discrete signals, so we need to S-transform discredited.

There is a relationship between the signal S transform and Fourier transform:

$$
S(\tau, f)=\int_{-\infty}^{\infty} H(\alpha+f) \exp \left(-\frac{2 \pi^{2} \alpha^{2}}{f^{2}}\right) \exp (j 2 \pi \alpha \tau) d \alpha \underset{f \neq 0}{ }
$$

If a discrete sequence of signals is $s(k T), k=1,2,3, \ldots N-1$, Sampling interval is $\mathrm{T}$, The total sampling time is $\mathrm{N}$. Wherein the sequence $s(k T)$ discrete Fourier transform is :

$$
H\left[\frac{n}{N T}\right]=\frac{1}{N} \sum_{k=0}^{N-1} h[k T] \exp \left(-j \frac{2 \pi n k}{N}\right) \quad n=0,1,2, \ldots, N-1
$$

The equation (7) into equation (6), and $f \rightarrow \frac{n}{N T}, \tau \rightarrow i T$, then S-transform can be converted to discrete form: 


$$
S\left[i T, \frac{n}{N T}\right]=\sum_{m=0}^{N-1} H\left[\frac{m+n}{N T}\right] \exp \left(-\frac{2 \pi^{2} m^{2}}{n^{2}}\right) \exp \left(j \frac{2 \pi m i}{N}\right)
$$

$$
i, m, n=0,1,2, \ldots N-1 \text {. }
$$

By the formula (6), (7), (8), we can see that discretization S-transform is a matrix. The value of an element of the matrix is the amplitude of a signal S-transform at the appropriate frequency and time. Column vector of the matrix represents the amplitude of the signal S-transform at the corresponding frequency. Row vector of the matrix, represents the amplitude of the signal S transform at the corresponding time. Then time-frequency image can be used to indicate the result of a signal Stransform.

\section{Detection Principle}

For discrete signals, if sequence $\left\{h_{k}, k=0,1,3, \ldots, n-1\right\}$ and sequence $\left\{H_{j}, j=0,1,2, \ldots, n-1\right\}$ constituting pair of the signal discrete Fourier transform, then the sum of the energy of the signal in the time domain is equal to the sum of the energy of the signal in the frequency domain.

$$
\sum_{k=0}^{n-1}\left|h_{k}\right|^{2}=\frac{1}{n} \sum_{j=0}^{n-1}|H j|^{2}
$$

In the above formula, the left side of the equation represents the energy in the time domain, the right side of the equation represents the energy in the frequency domain. For a power signal after $\mathrm{S}$ transform, that is, the signal from a time domain into a number of components in the frequency domain. Thus, Perceval theorem is applicable, i.e., the power signal after $\mathrm{S}$ transform, the energy is constant.

That is to say no matter what changes, as long as the original signal by a disturbance, into a $s^{\prime}(t)$, then the $S$ transform of the discretization of $S^{\prime}(t)$ can be expressed as $S^{\prime}(m, n)$, and in the matrix $S^{\prime}(m, n)$, at every moment, at the point where the disturbance occurred, the sum of all of its frequency amplitude changes. The $\mathrm{S}$ transform of the power system signal $\mathrm{s}(\mathrm{t})$ that has not been disturbed can be expressed as $S(m, n)$. The sum of the absolute value of all the elements of a column of the $S(m, n)$, namely the average energy within a sampling interval of the original signal.

As follows:

$$
\frac{1}{\mathrm{~T}} \int|s(t)|^{2} d t=\frac{1}{N} \sum_{k=0}^{N-1}\left|S\left(\tau, f_{k}\right)\right|^{2}
$$

The formula (10) is represented by the time-amplitude coordinate system. A power system signal $s(t)$ has not been disturbed, and its energy is displayed in the coordinate system is a straight line. Meanwhile, it can be seen from equation (10), when the signal subjected to frequency disturbances or amplitude disturbance, its average energy will change. The result of the $\mathrm{S}$ transform of a discrete power system signal subjected to disturbances can be expressed as $S^{\prime}(m, n)$, and its average energy within a sampling interval is changed compared with the formula (10).

I.e. after disturbed, the average energy of a signal within a sampling interval can be expressed as:

$$
\frac{1}{T} \int S^{\prime}(t)\left|d t=\frac{1}{N} \sum_{k=0}^{N-1}\right| S^{\prime}\left(\tau, f_{k}\right) \mid
$$

Then, for a power system signal that has been disturbed, its energy at the time-amplitude twodimensional coordinate system displayed certainly not a straight line. And disturbed at the beginning and end time, the energy curve will change, according to the disturbance signal is increased or decreased, the energy curve changes accordingly. Thus, according to this curve, it can locate the time 
of the power system disturbances. In order to accurately locate the disturbance, we deal with the average energy of the square; the energy curve will be sharper, i.e., more accurate positioning time.

Then, by the following equation of energy curve to accurately locate the disturbance moment, as shown below

$$
\left.\left.\frac{1}{\mathrm{~T}} \int\right|^{\prime}(t)\right|^{4} d t=\frac{1}{N} \sum_{k=0}^{N-1}\left|s^{\prime}\left(\tau, f_{k}\right)\right|^{4}
$$

The matrix elements of all rows are added, then the frequency as the horizontal axis coordinate diagram, you can be get each frequency component of a signal.

\section{The detection simulation of a transient power voltage disturbance signal based on $\mathrm{S}$ transform}

The simulation used in transient power quality disturbance signal, generated using MATLAB. This paper focuses on several common disturbance signal simulation and analysis. In the simulation, the sampling frequency for each disturbance signal is set to $1000 \mathrm{~Hz}$. Since the signal studied in this paper from the power system, thus setting all the fundamental frequency of the disturbance signal is $50 \mathrm{~Hz}$. wherein the sampling frequency of the high frequency oscillation is $30 \mathrm{kHz}$. Taking into account the presence of white noise signals in grid ${ }^{[7]}$, and therefore, all the signals used in the simulation herein, also a certain amount of white noise is added, so to simulate more realistic.

A. The simulation results and analysis of voltage sag

Voltage sag of the original signal can be expressed as

$$
\mathrm{y}=\left[1-\mathrm{k}\left(\mathrm{t}_{1}<\mathrm{t}<\mathrm{t}_{2}\right)\right] \sin \left(\omega_{0} t\right)
$$

$\mathrm{k}$ beyond a specific time interval, always be zero, and $\mathrm{k}$ is typically between 0.1 to 0.9 . Within the time interval ${ }^{\mathrm{t}_{1}<\mathrm{t}<\mathrm{t} 2}$, different values of $\mathrm{k}$, to determine the different voltage sag signal to be simulated. The simulations are shown in Figure .1-3.

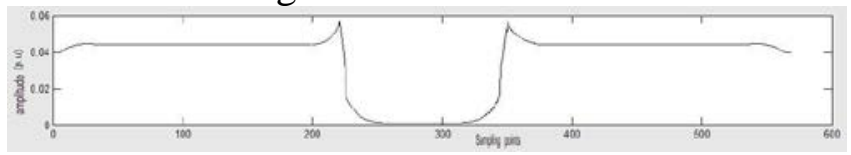

Figure1. The curve of the sum of amplitude square

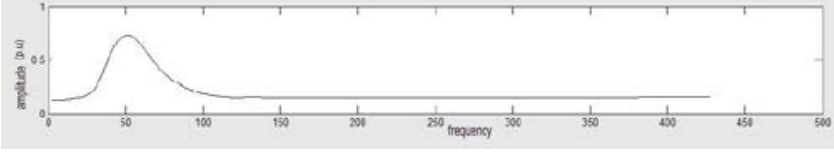

Figure 2.Frequency - amplitude curve

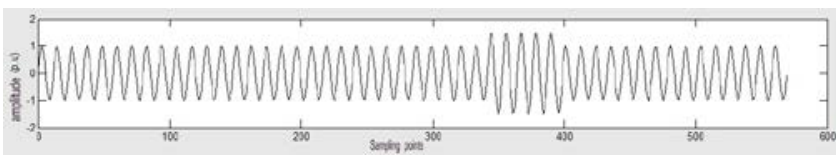

Figure 3.Voltage swell waveforms

As can be seen from the simulation Figure.2, the curve of the sum of the amplitude square of a signal transformed by S-transform will have sharp peaks at occurrence and end time of the voltage sag. Detecting the time of occurrence of these two peaks, the disturbance can be accurately positioned. Figure. 3 shows the value of the frequency of the signal at $50 \mathrm{~Hz}$ is largest, that is the main component of the signal is a frequency signal of $50 \mathrm{~Hz}$.

B. The simulation results and analysis of voltage swell

Voltage swell of the original signal can be expressed as

$$
\mathrm{y}=\left[1+\mathrm{k}\left(\mathrm{t}_{1}<\mathrm{t}<\mathrm{t}_{2}\right)\right] \sin \left(\omega_{0} t\right)
$$


$\mathrm{k}$ beyond a specific time interval always is zero, and $\mathrm{k}$ is typically between 0.1 to 0.9 . Within the time interval ${ }^{t_{1}<t<t_{2}}$, different values of $k$, to determine the different voltage sag signal to be simulated. The simulations are shown in Figure .4-5

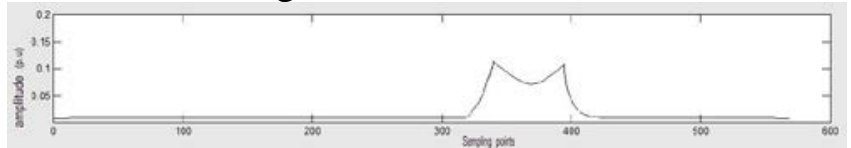

Figure4.The curve of the sum of amplitude square

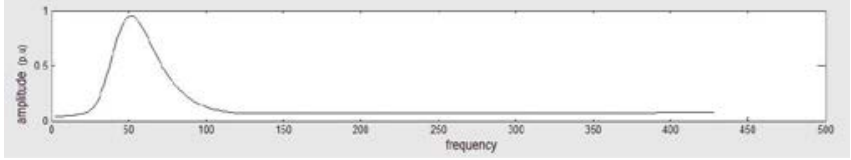

Figure5. requency - amplitude curve

As can be seen from the simulation Figure.5, the curve of the sum of the amplitude square of a signal transformed by S-transform will have sharp peaks at occurrence and end time of the voltage swell. Detecting the time of occurrence of these two peaks, the disturbance can be accurately positioned. Figure. 6 shows the value of the frequency of the signal at $50 \mathrm{~Hz}$ is largest, that is the main component of the signal is a frequency signal of $50 \mathrm{~Hz}$.

\section{Conclusion}

This paper presents a disturbance localization algorithm, based on the sum of the amplitude square of a signal transformed by S-transform. MATLAB simulation shows that this algorithm has a unique advantage in the analysis of non-stationary signals have transient, mutation and other characteristics, and will have a certain prospect in the detection and identification of the power quality disturbances.

\section{Acknowledgements}

The research work was supported by the technology project of State Grid Shanxi Electric Power Company named the dynamic monitoring technology research and application of wires carrying capacity based on parameter identification and Key Projects in the National Science \& Technology Pillar Program under Grant No. 2012BAA15G00.

\section{References}

[1] Heydt G T, Fjeld P S, Liu C C. "Applications of the window FFT to electic power quality assessment”, IEEE Trans.on Power Delivery, 1999, 14(3): 1411-1416.

[2] Math H J, Bollen. "Understanding power quality problems: voltage sags and interruptions”, IEEE, Inc, New York, 2000: 139-253.

[3] Xiao Xiangning, Han Minxiao,Xu Yonghai. Power Quality Analysis and Control [M],Beijing: China Electric Power Press,2004,21-33.

[4] Gargoom Ameen, Ertugrul Nesimi, Soong W L. "Power quality indices measurement using the S-transform”, International Journal of Power and Energy Conversion, 2009, 1(1) :31-48.

[5] O Poisson, M Meullier. "New signal processing tools applied to power analysis". IEEE Transactions on Power Delivery, 1999, 14(2): 56l-566.

[6] Emmanouil Styvaktakis, Math H J Bollen, Irene Y H Gu. "Classification of power system transients: synchronised switching”, Pmceedings of IEEE Power Engineering Sociely Winter Meetmg, Singapore, 2000, V01.4: 268l-2686.

[7] Miaden, Kezunovic. "Automated analysis of voltages sags, their causes and impacts”, Proceedings of IEEE Power Engineering Society Summer Meeting, Vancouver, BC Canada, 2001, V012: 1113-1117. 